\title{
APPLICATION OF QUANTILE REGRESSION TO ASYMMETRIC PRICE TRANSMISSION ESTIMATION: INSIGHTS FROM MONTE CARLO SIMULATIONS
}

\author{
Acquah De-Graft H., Associate Professor \\ Department of Agricultural Economics and Extension, University of Cape Coast, \\ Cape Coast, Ghana \\ E-mail: henrydegraftacquah@yahoo.com
}

\begin{abstract}
Paper introduces and compares performances of Quantile regression approach to the conventional Ordinary Least Squares methods for estimation of asymmetric price transmission model when the true data generating process is known. Monte Carlo simulation results indicate that the estimates of the coefficients of the asymmetric price transmission model derived from the Least squares and the Quantile regression approaches are accurate and equivalent or close to their true values for normal data regardless of variability in sample size. Least squares method is affected by outliers and yields inaccurate estimates of the coefficients of the asymmetric price transmission model across various sample sizes when the data contains outliers. Quantile regression estimation remains robust to outliers in large samples and provides estimates of the coefficients of the asymmetric price transmission model that are accurate and nearly equivalent to their true values. The evidence from Monte Carlo experimentation suggests that the proposed Quantile regression estimation is likely to do no worse than the OLS with normal dataset and promise to do better when the dataset has outliers within the asymmetric price transmission modelling context.
\end{abstract}

\section{KEY WORDS}

Monte Carlo Simulation, Quantile Regression Estimation, Granger and Lee Asymmetry, Ordinary Least Squares Estimation, outlier.

Over the years data used in measuring price asymmetry has been found to contain outliers. For example, some studies (Kind (2015), Douglas (2010) and Karfakis and Rapsomanikis (2007)) have found outliers in data used in price transmission analysis. Douglas (2010) investigated price asymmetry and found that the presence of outliers in price data generated evidence of spurious asymmetry. Kind (2015) also notes that data used in agricultural price analysis from developing countries are more often found to have outliers and as result, estimates obtained from such contaminated data lose their value. Karfakis and Rapsomanikis (2007) also noted in a spatial price analysis of selected agricultural commodities, that the error correction modelling methods are not robust to the presence of outliers in the price series. In effect, a common problem in price transmission regression analysis is the presence of outliers. Barnett and Lewis (1994) defined outliers as observations that appear inconsistent with the rest of data. Outliers may occur as a result of unusual but explainable events, such as faulty measurement, incorrect recording of data, failure of a measurement instrument, etc.

Though the conditional mean models such as the ordinary least squares (OLS) have been used extensively in asymmetric price transmission analysis, they have some limitations. Assumptions related to the random errors are not always satisfied in reality. The least squares regression model gives misleading results in the presence of outliers and may produce wrong estimates of the asymmetric adjustment coefficient, which provides the basis for detecting price asymmetry. In effect, erroneous conclusions will be made from the asymmetric price transmission model when the regular assumptions of the OLS are not met or when the data contains outliers.

An alternative approach to estimate asymmetric price transmission whilst concurrently accommodating outliers in the data is to employ Quantile regression approach. Quantile regression remains robust to outliers and has been met with success in estimation of linear 
models in the presence of outliers as detailed in Koenker and Hallock (2001); Fox \& Weisberg (2010); Bancayrin-Baguio (2009); and Julali and Babanezhad (2011). Though previous studies addressing outlier problems in asymmetric price transmission analysis have employed M-estimators (Acquah, 2017a) and Rank -based estimation (Acquah, 2017b), no studies has considered the use of the quantile regression approach in asymmetric price transmission estimation when the price data contains outliers.

Empirically, very little is known about the relative performance of Quantile regression and the OLS method for estimation of asymmetric price transmission models when the data contains outliers. To explore this issue, Monte Carlo methods are employed to investigate the performance of the OLS and Quantile regression in estimating the Granger and Lee asymmetry using data with and without outliers.

The paper is structured as follows. The introduction is followed by the methods section which discusses the Granger and Lee Asymmetric model, Ordinary Least Squares (OLS) method and Quantile Regression approach. The results and discussion present a practical application in which the performance of the OLS and Quantile Regression approach in estimating true values of the Granger and Lee asymmetric data generating process is evaluated and the results of the Monte Carlo simulations are presented. Finally, the study ends with a conclusion.

\section{METHODS OF RESEARCH}

Granger and Lee Asymmetry. To model asymmetric price transmission between two variables $x$ and $y$ integrated of the order one processes that are cointegrated the Granger and Lee (1989) Asymmetric Error Correction Model data generating process can be specified as follows:

$$
\Delta y_{t}=\beta_{1} \Delta x_{t}+\beta_{2}^{+}(y-x)_{t-1}^{+}+\beta_{2}^{-}(y-x)_{t-1}^{-}+\varepsilon_{1, t} \varepsilon_{1, t} \sim N\left(0, \delta^{2}\right)
$$

The long run equilibrium relationship between $y$ and $x$ is captured by a symmetric error correction term $(y-x)$. Asymmetric adjustments can be introduced by segmentation of the error correction term into positive and negative components as follows:

$$
\begin{aligned}
& (y-x)_{t}^{+}=\left[\begin{array}{c}
(y-x)_{t}, \text { if }(y-x)_{t}>0 \\
\text { zero otherwise }
\end{array}\right. \\
& (y-x)_{t}^{-}=\left[\begin{array}{c}
(y-x)_{t}, \text { if }(y-x)_{t}<0 \\
\text { zero otherwise }
\end{array}\right.
\end{aligned}
$$

In order to incorporate asymmetric adjustments effects, the speeds of adjustments are allowed to differ for the positive and negative components of the Error Correction Term (ECT) since the equilibrium relationship captured by the ECT is symmetric. The test for symmetry in eq. (1) is conducted by determining whether the coefficients $\left(\beta_{2}{ }^{+}\right.$and $\left.\beta_{2}{ }^{-}\right)$are identical (that is $H_{0}: \beta_{2}^{+}=\beta_{2}^{-}$). The Granger and Lee Asymmetric Error correction model in eq. (1) can be considered as a standard regression model and the estimation of its parameters can be done using Ordinary Least Squares method and Quantile Regression technique. Empirical results are computed for the two techniques and compared.

Ordinary Least Squares Estimation (OLS). The OLS is a technique for fitting the best straight line by minimizing the sum of squared errors. In the OLS a squared error loss function, $l(e)=\varepsilon^{2}$, is employed. Estimate of the conditional mean of the dependent variable can be obtained by minimizing the sum of squared residuals. When the conditional mean is linear in $x, E[y \mid x]=x^{\prime} \beta$, the sample mean $u$ solves: 


$$
\arg \min \sum_{i=1}^{n}\left(y_{i}-u\right)^{2}
$$

The sample mean can be used to obtain the unconditional population mean by replacing $u$ with $x^{\prime} \beta$ and solving:

$$
\arg \min \sum_{i=1}^{n}\left(y_{i}-x^{\prime} \beta\right)^{2}
$$

Quantile Regression. Quantile regression was first introduced by Koenker and Bassett (1978; 1982) as a robust alternative to least-squares regression. In quantile regression, quantiles of the conditional distribution of the dependent variable are expressed as a function of observed independent variables. Quantile regression offers a number of advantages over least-squares methods. For example, quantile regression does not require the restrictive assumptions of least-squares regression (assumes that the error terms are normally distributed with a mean of zero and a constant variance). Furthermore, since quantile regression estimates quantiles of the conditional distribution rather than the mean, it is more resistant to outliers than least-squares methods (Leider, 2012). The linear quantile regression model is given by the conditional quantile functions (indexed by the quantile $\tau$ ).

$$
Q_{y}(\tau \mid x)=x_{i}^{T} \beta
$$

$Q_{y}(\tau \mid x)$ denotes the $\tau$-quantile of $y$ conditional on $x$. Estimates are obtained by minimizing $\sum_{i} \rho_{\tau}\left(y_{i}-x_{i}^{T} \beta\right)$ with respect to $\beta$ where for $\tau \in(0,1), \rho_{\tau}$ denotes the pairwise linear function $\rho_{\tau}(u)=u\{\tau-I(u<0)\}, I$ being the indicator function. This is a linear programing problem. Estimating the quantile regression at $\tau=0.5$, gives the special case of the median regression which is implemented in this study.

\section{RESULTS AND DISCUSSION}

Quantile Regression and Ordinary Least Squares for Estimation of Price Asymmetry. The true data generating process in the context of the Granger and Lee asymmetric error correction model can be specified as follows:

$$
\Delta y_{t}=0.7+0.5 \Delta x_{t}-0.25\left(y_{t}-x_{t}\right)^{+}{ }_{t-1}-0.75\left(y_{t}-x_{t}\right)^{-}{ }_{t-1}+\varepsilon
$$

The variables $y_{t}$ and $x_{t}$ are are non-stationary variables that are integrated of the order one. A cointegrating relationship exists between $\mathrm{y}$ and $\mathrm{x}$ which is defined by the error correction term $\left(y_{t}-x_{t}\right)_{t-1}$. The positive and negative components of the error correction term are represented by $\left(y_{t}-x_{t}\right)^{+}{ }_{t-1}$ and $\left(y_{t}-x_{t}\right)^{-}{ }_{t-1}$. The errors are normally distributed with a mean 0 and a variance of $1[\varepsilon \square N(0,1)]$ for normal data. In order to create data with outliers, nine observations of the errors generated for the normal data with values generated from the normal distribution with a mean of 0 and a variance of 1 is replaced with nine observations from the normal distribution with a mean of 20 and variance of $1(\varepsilon \square N(20,1))$.

In order to investigate the performance of the Quantile regression $(\tau=0.5)$ and OLS in estimating the true values of the asymmetric price transmission model, 1000 regressions based on the Granger and Lee model specified in eq. (7) is estimated. The Monte Carlo studies are conducted under conditions of different sample sizes $(50,150$ and 500$)$ and 
asymmetry given by $\left(\beta_{2}^{+}, \beta_{2}^{-}\right) \in(-0.25,-0.75)$ for the normal data as well as the data with outliers. This study draws from Cook et al $(1999,2000)$ and Acquah $(2012,2013)$ to assign the asymmetric adjustment parameters $\left(\beta_{2}^{+}, \beta_{2}^{-}\right)$.

The results derived from the Monte Carlo experimentation for the normal data are reported in Table 1. Results of 1000 Monte Carlo simulations indicate that the estimates of the coefficients of the asymmetric price transmission model obtained from the Quantile regression analysis are accurate and close to their true parameter values for the data without outliers (Normal data) with small and moderate sample sizes (50 and 150). The estimates of the coefficients of the asymmetric price transmission model derived from the least squares methods are accurate and equal to their true parameter values for the data without outliers (Normal data) with small and moderate sample sizes (50 and 150). Notably, the estimates of the coefficients of the asymmetric price transmission model derived from the Least squares method and the Quantile Regression analysis are accurate and equivalent to their true parameter values for normal data with large sample size (500).

In summary, Table 1 demonstrates that in the absence of outliers, the OLS and Quantile regression analysis performed well, with the averaged estimates all nearly equivalent or close to their true values of $\beta_{0}=0.7, \beta_{1}=0.5, \beta_{2}=-0.25, \beta_{3}=-0.75$ regardless of the different sample sizes. These results are consistent with Ryan (1997) who noted that robust regression estimation technique performs almost as well as OLS when the data has no outliers.

Table 1 - Normal Data (Without Outliers)

\begin{tabular}{|c|c|c|c|c|c|c|}
\hline \multirow{2}{*}{ Sample Size } & \multirow{2}{*}{ Properties of Data } & \multirow{4}{*}{ Method } & \multicolumn{4}{|c|}{ Estimates } \\
\cline { 3 - 7 } & & & $\beta_{0}$ & $\beta_{1}$ & $\beta_{2}$ & $\beta_{3}$ \\
\hline \multirow{2}{*}{$\mathrm{N}=50$} & \multirow{2}{*}{ Normal } & OLS & 0.70 & 0.50 & -0.25 & -0.75 \\
\cline { 3 - 7 } & \multirow{2}{*}{ Normal } & Quantile Regression & 0.70 & 0.50 & -0.25 & -0.75 \\
\cline { 3 - 7 } & & OLS & 0.70 & 0.50 & -0.25 & -0.75 \\
\cline { 3 - 7 } & \multirow{2}{*}{$N=150$} & Quantile Regression & 0.70 & 0.50 & -0.25 & -0.74 \\
\hline \multirow{2}{*}{$\mathrm{N}=500$} & \multirow{2}{*}{ Normal } & OLS & 0.70 & 0.50 & -0.25 & -0.75 \\
\cline { 3 - 7 } & & Quantile Regression & 0.70 & 0.50 & -0.25 & -0.75 \\
\hline
\end{tabular}

Based on 1000 Monte Carlo Simulation.

The results derived from the Monte Carlo experimentation for the data with outliers are reported in Table 2. Results of 1000 Monte Carlo simulations indicate that the estimates of the coefficients of the asymmetric price transmission model derived from the Quantile regression analysis are accurate and close to their true parameter values for the data with outliers in large sample (500). Generally, as sample size increase from small through moderate to large sample, estimated coefficients of the asymmetric price transmission model move closer to their true parameter values in the Quantile regression analysis.

In the presence of outliers, the ordinary least squares method performed poorly as illustrated in Table 2. In small, moderate and large samples of 50, 150 and 500 respectively, the ordinary least squares (OLS) estimator performs poorly with its parameter estimates entirely different from the true parameter values of $\beta_{0}=0.7, \beta_{1}=0.5, \beta_{2}=-0.25, \beta_{3}=-0.75$ as specified in the data generating process. This is consistent with Chatterjee and Hadi (1986) who noted that outliers may have a marked influence on parameter estimates in linear regression analysis.

The foregoing discussion points to the fact that the results of the quantile regression analysis are similar to that of the ordinary least squares and close to their true values when the data contains no outliers. However, when the data contains outliers, the least squares is affected by outliers in small, moderate and large samples whilst the quantile regression analysis remains robust to outliers in large samples.

The results are consistent with Fox and Weisberg (2010) assertion that robust methods such as Quantile regression estimation methods perform much better than OLS when the 
data has outliers. Similarly, Bancayrin-Baguio (2009) note that quantile regression estimation technique gives better asymptotic efficient estimates for the coefficients of the regression model than the least squares method.

Table 2 - Data with Outliers

\begin{tabular}{|c|c|c|c|c|c|c|}
\hline \multirow{2}{*}{ Sample Size } & \multirow{2}{*}{ Properties of Data } & \multirow{4}{*}{ Method } & \multicolumn{4}{|c|}{ Estimates } \\
\cline { 3 - 6 } & & & $\beta_{0}$ & $\beta_{1}$ & $\beta_{2}$ & $\beta_{3}$ \\
\hline \multirow{2}{*}{$\mathrm{N}=50$} & \multirow{2}{*}{ With Outliers } & OLS & 3.04 & 0.50 & -0.16 & -1.08 \\
\cline { 3 - 7 } & \multirow{2}{*}{$\mathrm{N}=150$} & Wuantile Regression & 0.87 & 0.50 & -0.24 & -0.77 \\
\hline \multirow{2}{*}{$\mathrm{N}=500$} & \multirow{2}{*}{ With Outliers } & OLS & 1.00 & 0.47 & -0.44 & -2.01 \\
\cline { 3 - 7 } & & Quantile Regression & 0.72 & 0.50 & -0.26 & -0.83 \\
\cline { 3 - 7 } & & OLS & 1.00 & 0.50 & -0.26 & -0.52 \\
\hline
\end{tabular}

Based on 1000 Monte Carlo Simulation.

Furthermore, the results are consistent with additional studies (Julali and Babanezhad (2011), Koenker and Hallock (2001) and Koenker and Bassett (1978; 1982) which assert that the quantile regression is a robust alternative to the least squares method when the data contains outliers.

\section{CONCLUSION}

The performance of quantile regression analysis have been investigated in asymmetric price transmission regression modelling. The findings suggest that the quantile regression approach yield similar results as the OLS with normal data. However, when outliers are present in the data, the least squares does not provide accurate estimates of the coefficients of the true asymmetric price transmission model in small, moderate and large samples of data. Quantile regression estimation, on the other hand, is robust and provides precise estimates of the coefficients of the true asymmetric price transmission model in large samples. The results of the Monte Carlo simulation indicate that the Quantile regression estimation can be considered an alternative to the OLS technique in asymmetric price transmission estimation and may yield accurate results in large samples when the data contains outliers.

\section{REFERENCES}

1. Acquah, H. D. (2017a). M-Estimators for Asymmetric Price Transmission Modelling. Russian Journal of Agricultural and Socio-Economic Sciences, 2(62), 12-19.

2. Acquah, H. D. (2017b). Rank-Based Estimation for Asymmetric Price Transmission Modelling. Russian Journal of Agricultural and Socio-Economic Sciences, 3(63), 86-92.

3. Acquah, H. D. (2013). Using bootstrap method to evaluate the power of tests for nonlinearity in asymmetric price relationship. Journal of Economics and Behavioral Studies, 5, (4), 237-241.

4. Acquah, H. D. (2012). A bootstrap approach to testing for symmetry in the Granger and Lee Asymmetric Error Correction Model. Russian Journal of Agricultural and SocioEconomic Sciences, 11(11), 33-36.

5. Bancayrin-Baguio, C. (2009). Performance of Median and Least Square Regression for Slightly Skewed Data. World Academy of Sciences, Engineering and Technology $p 53$

6. Barnett, V. \& Lewis, T. (1994) Outliers in Statistical Data, 3rd Edition, Wiley, New York, USA.

7. Chatterjee, S. \& Hadi, A.S. (1986) Influential observations, high leverage points, and outliers in linear regression, Statistical Science, 1, 379-393.

8. Cook, S., Holly, S., \& Turner, P. (1999). The Power of tests for non-linearity: the case of Granger-Lee asymmetry, Economics Letters, 62, pp.155-159. 
9. Cook, S., Holly, S., \& Turner, P. (2000). The Power of Tests for Non-linearity: The Escribano-Pfann Model, Computational Economics, 15, pp. 223-226.

10. Douglas, C. C. (2010). Do gasoline prices exhibit asymmetry? Not usually!. Energy Economics, 32(4), 918-925.

11. Fox, J. \&Weisberg S. (2010) Robust Regression in R, An Appendix to an R companion to Applied Regression, Second Edition

12. Granger, C. W. J., \& Lee, T. H. (1989). Investigation of production, sales and inventory relationships using multicointegration and non-symmetric error correction models. Journal of applied econometrics, 4(S1), S145-S159.

13. Jalali, N. \& Babanzhad, M. (2011) Quantile Regression due to Skewness and Outliers. Applied Mathematics Sciences, Vol 5 no.39 1947-1951.

14. Karfakis, P., \& Rapsomanikis, G. (2007). Margins across time and space: threshold cointegration and spatial pricing applications to commodity markets in Tanzania. In workshop on staple food trade and market policy options for promoting development in eastern and southern Africa.

15. Kind, M (2015). Analysis of Market Integration- An Alternative Approach. MSc Thesis submitted to the Agricultural Economics and Rural Policy Group, Wageningen University, Netherland.

16. Koenker, R. \& Hallock, K.F. (2001) Quantile Regression. Journal of Economic Perspectives- volume 15, Number 4, pages 143-156

17. Koenker, R. \& Bassett, G. (1982). Robust tests for heteroscedasticity based on regression quantiles. Econometrica, 50, 43-61.

18. Koenker, R. \& Bassett, G. (1978). Regression Quantiles. Econometrica, 46, 33-50.

19. Leider, J. (2012). A Quantile Regression Study of Climate Change in Chicago, 19602010. Department of Mathematics, Statistics and Computer Science, University of Illinois, Chicago.

20. Ryan, T. P. (1997). Modern regression methods. New York, NY: John Wiley \& Sons, Inc. 